\title{
REASSESSMENT OF THE CATALYTIC ACTIVITY AND SUBSTRATE SPECIFICITY OF FKBP35 FROM Plasmodium knowlesi USING PROTEASE-FREE ASSAY
}

\section{Cahyo Budiman ${ }^{*}$, Carlmond Goh Kah Wun', Lee Ping Chin², Rafida Razali', Thean Chor Leow ${ }^{3}$}

\begin{abstract}
'Biotechnology Research Institute, Universiti Malaysia Sabah, Kota Kinabalu Sabah, Malaysia ${ }^{2}$ Faculty of Science and Natural Resources, Universiti Malaysia Sabah, Kota Kinabalu Sabah, Malaysia ${ }^{3}$ Enzyme and Microbial Technology Research Center, Department of Cell and Molecular Biology, Faculty of Biotechnology and Biomolecular Sciences, Universiti Putra Malaysia, Serdang, Selangor, Malaysia *Corresponding author's email: cahyo@ums.edu.my Received date: 1 September 2020 | Accepted date: 21 September 2020
\end{abstract}

\section{ABSTRACT}

FK506-binding protein35 of Plasmodium knowlesi (Pk-FKBP35) is a member of peptidyl prolyl cis-trans isomerase (PPlase) and is considered as a promising avenue of antimalarial drug target development. This protein is organized into the $\mathrm{N}$-terminal domain responsible for PPlase catalytic activity followed and the tetratricopeptide repeat domain for its dimerization. The protease-coupling and protease-free assays are known to be the common methods for investigating the catalytic properties of PPlase. Earlier, the protease-coupling assay was used to confirm the catalytic activity of Pk-FKBP35 in accelerating cis-trans isomerization of the peptide substrate. This report is aimed to re-assess the catalytic and substrate specificity of Pk-FKBP35 using an alternative method of a protease-free assay. The result indicated that while Pk-FKBP35 theoretically contained many possible cleavage sites of chymotrypsin, experimentally, the catalytic domain was relatively stable from chymotrypsin. Furthermore, under protease-free assay, Pk-FKBP35 also demonstrated remarkable PPlase catalytic activity with $k_{\text {cat }} / K_{\mathrm{M}}$ of $4.5 \pm 0.13 \times 10^{5} \mathrm{M}^{-1} \mathrm{~s}^{-1}$, while the $k_{\text {cat }} / K_{\mathrm{M}}$ of active site mutant of $\mathrm{D} 55 \mathrm{~A}$ is $0.81 \pm 0.05 \times 10^{5} \mathrm{M}^{-1} \mathrm{~s}^{-1}$. These values were considered comparable to $k_{\mathrm{cat}} / K_{\mathrm{M}}$ obtained from the protease-coupling assay. Interestingly, the substrate specificities 
of Pk-FKBP35 obtained from both methods are also similar, with the preference of PkFKBP35 towards Xaa at P1 position was Leu $>$ Phe $>$ Lys $>$ Trp $>$ Val $>$ Ile $>$ His $>$ Asp $>$ Ala $>$ Gl $\mathrm{n}>\mathrm{Glu}$. Altogether, we proposed that protease-free and protease-coupling assays are reliable for Pk-FKBP35.

Keywords: malaria, Plasmodium knowlesi, Peptidyl prolyl cis-trans isomerases, FKBP

\section{INTRODUCTION}

Plasmodium knowlesi is known as the fifth species of Plasmodium, causing malaria in humans through a long tail macaque (Singh et al., 2004; Sing \& Daneshvar, 2013). Statistically, Malaysia is considered the epicentre of Plasmodium knowlesi malaria, which accounts for $90 \%$ of human malaria cases. A recent report showed that more than 7,000 of $P$. knowlesi infection cases were reported in Malaysia, $86.8 \%$ of which were detected in Malaysian Borneo (Divis et al., 2020). In Sabah, P. knowlesi infection cases were scattered all regions of Sabah, including Tawau, interior division, West Coast division, Sandakan division, and also Kudat division (Lau, Joveen-Neoh, \& Chong, 2011; Goh et al., 2013; William et al., 2014). Rajharam et al. (2019) also reported 6 malaria deaths in Sabah during 2015 - 2017, all were from P. knowlesi. Altogether, this demonstrates that $P$. knowlesi remains a severe issue in Sabah and urges more collective attempts to fight it.

The genome sequence of $P$. knowlesi harbours a single gene encoding a 35 kDa FK506binding protein (Pk-FKBP35), a member of peptidyl prolyl cis-trans isomerase (PPlase) family proteins. PPlase is a group of enzymes capable of catalysing slow isomerization of a cis-proline bond which is known to be a rate-limiting step of protein folding. It is therefore predicted that this protein plays a vital role in the cellular function of $P$. knowlesi due to its involvement in protein folding machinery of the parasite. Hariskishore et al. (2014) proposed that Plasmodium FKBP35 is indeed a viable target for the development of novel antimalarial drugs. Accordingly, comprehensive structure and functional studies, as well as attempts to find inhibitors targeting this protein were widely reported (Yoon et al., 2006; Hariskishore et al., 2014; Goh et al., 2018). This protein is a dimeric protein in solution with the size of about $78 \mathrm{kDa}$ and structurally organized into two functional domains of the N-terminal FK506-binding domain (FKBD) and tetratricopeptide repeat (TPR) domain.

The study on the kinetics of a reaction catalysed by the enzyme targeted by the drug is an important avenue for further drug development. Accordingly, extensive studies were reported on the catalytic properties of Plasmodium FKBP35, with the aim is to understand the mechanism behind the catalysis as a platform for the discovery 
of drug-able spots for inhibiting the catalysis. Two approaches were reported to determine the catalytic activity of PPlase family members: protease-coupling and protease-free assays. In the protease-coupling assay, chymotrypsin was used as the protease and synthetic prolyl bond containing tetrapeptide conjugated with fluorophore moiety assay relies on the released of $p$-nitroaniline from the substrate due to cleavage by chymotrypsin when the proline residue in the substrate assumes the trans conformation. The cleavage has not occurred when the prolyl bond is in the cis conformation. Accordingly, the increase in isomerization is implicit in the increased $p$-nitroaniline release rate because catalysis of isomerization produces trans substrate with increased frequency. While this method is widely used in the measurement of catalytic activity of PPlase, a major drawback of this method is the possibility of PPlase degradation by chymotrypsin used in the assay.

On the other hand, an alternative method of uncoupled PPlase assay was proposed by Janowski, Wöllner, Schutkowski, and Fischer (1997), which omitted the use of any protease and relied on cis prolyl bond changes conformers upon a solvent jumping event. The solvent jumping is based on the ability of lithium chloride ( $\mathrm{LiCl}$ ) in anhydrous tetrahydrofuran (THF) or trifluoroethanol (TFE) to shift the equilibrium between cis and trans of Xaa-Pro peptide bonds in favour of the cis conformer. Under a normal solvent, the cis prolyl bond population is about only $10-20 \%$ at the equilibrium state. This population is extremely increased when the solvent changed to $\mathrm{LiCl}$ in TFE or THF to $80-90 \%$ (Kofron et al., 1992). The absence of protease in the uncoupling method also provides an opportunity to have more reliable kinetic parameters of the isomerization and substrate specificity. In the protease-coupling assay, the kinetic parameters and substrate specificity of PPlase might be intersected by the protease due to the possibility of the protease in binding and degrade the substrate during the isomerization. While the limitation of the protease-free assay lies in the high noise of the spectroscopic signal between cis and trans isomers, this method is recommended for the multidomain PPlase and sensitive towards chymotrypsin.

Earlier, catalytic properties of Pk-FKBP35 were studied using a protease-coupling method, which indicated that FKBD acted as the catalytic domain for this protein, and TPR has no role in the catalytic activity (Goh et al., 2018). Therefore, it is interesting if the catalytic activity of Pk-FKBP35 under protease-coupling assay reflects its real activity without the interference of chymotrypsin. Accordingly, in this report, the sensitivity of Pk-FKBP35 towards chymotrypsin protease was confirmed. Reassessment of the catalytic activity and substrate specificity of Pk-FKBP35 using the protease-free assay was then discussed and compared to those obtained using the protease-coupling assay. 


\section{MATERIALS AND METHODS}

\section{Protein Preparation}

All proteins were prepared in a His-tagged form. The expression constructs of $p E T 29$ Pk-FKBP35 was used to overproduce full-length Pk-FKBP35. Meanwhile, the constructs of pET29-D55A were used to overproduce active site mutants of D55A, where a single amino acid of Asp55 was replaced by Ala. All constructs were obtained as previously reported (Goh et al., 2018) and transformed into Escherichia coli BL21(DE3) for overproduction purposes.

\section{Overproduction and Purification}

Overproduction and purification of the proteins were carried out as described previously (Goh et al., 2018). Expression and purity of Pk-FKBP35 and D55A were checked by SDS-PAGE using 15\% polyacrylamide gel stained under Coomassie Brilliant Blue R250 (Laemmli, 1970). The UV absorption at $280 \mathrm{~nm}$ was used to measure the protein concentration with the extinction coefficient at $280 \mathrm{~nm}$ of a $0.1 \%\left(1 \mathrm{mg} \mathrm{mL}^{-1}\right)$ is 0.73 for Pk-FKBP35 and D55A which was calculated based on Goodwin and Morton (1946).

\section{Stability of Pk-FKBP35 against Chymotrypsin}

In silico prediction. The amino acid sequence of Pk-FKBP35 was firstly retrieved from PlasmoDB.org (accession code: PK-NH_1467100). The sequence was then subjected to PeptideCutter (https://web.expasy.org/peptide_cutter/). Chymotrypsin-low specificity (C-terminal to $[\mathrm{FYWML}]$, not before $\mathrm{P}$ ) and chymotrypsin-high specificity (C-terminal to [FYW], not before P) were selected as the proteases to digest. The display of cleavage sites was set as the default.

Limited proteolysis assay. Pk-FKBP35 was prepared in 35 mM HEPES buffer pH 7.8 at a final concentration of $0.5 \mathrm{mg} \mathrm{mL}^{-1}$. This solution was then incubated at $25^{\circ} \mathrm{C}$ for $3 \mathrm{~min}$. The chymotrypsin was then added into the solution at the final concentration of $15 \mu \mathrm{M}$. The mixture was then incubated at 10,20 and $30 \mathrm{~min}$ at $25^{\circ} \mathrm{C}$. The reaction was stopped by the addition of $0.5 \mathrm{mM}$ phenylmethylsulfonyl fluoride (PMSF). The proteins were then visualized and analyzed by using 15\% SDS-PAGE (Laemmli, 1970) using a $15 \%$ polyacrylamide gel, followed by staining with Coomassie Brilliant Blue. The concentrations of Pk-FKBP35 and chymotrypsin were higher than the assay conditions for the visibility of the proteins in SDS-PAGE. 
The $\mathbf{N}$ terminal amino acid sequencing. The gel obtained from limited protease assay as obtained above was transferred electrophoretically to polyvinylidenedifluoride (PVDF) membrane followed by staining and destaining using $0.5 \%$ Coomassie blue R-250 and $50 \%$ methanol, respectively. The membrane was then dried at $37^{\circ} \mathrm{C}$ and the bands of interest were excised gently and placed individually in sterilized 1.5 $\mathrm{mL}$ Eppendorf tube, treated with $100 \mu \mathrm{l}$ anhydrous trifluoroacetic acid (TFA) and incubated for $1 \mathrm{~h}$ at $40^{\circ} \mathrm{C}$ followed by evaporation. The PVDF membrane carrying the bands of interest were then further treated with $100 \mu \mathrm{l}$ of $0.6 \mathrm{~N} \mathrm{HCl}$ at $25^{\circ} \mathrm{C}$ for $12 \mathrm{~h}$. The sequencing of $\mathrm{N}$-terminal amino acids was carried out using Edman degradation with a Procise 491 protein sequencer (Applied Biosystems).

\section{PPlase Catalytic Activity}

The PPlase catalytic activity was determined by protease-free assay at $25^{\circ} \mathrm{C}$ according to previous reports (Janowski et al., 1997; Budiman, Tadokoro, Angkawidjaja, Koga, \& Kanaya, 2012). Briefly, the enzyme was incubated in $2 \mathrm{~mL}$ of $35 \mathrm{mM} \mathrm{HEPES}$ buffer (pH 7.8) for 3 min prior to the addition of the substrate. The peptide substrate suc-ALPFpNA (Wako Pure Chemical Industries, Osaka, Japan) was dissolved in anhydrous TFE containing $0.5 \mathrm{mM} \mathrm{LiCl}$. The reaction was initiated by the addition of $0.25 \mu \mathrm{M}$ sucALPF-pNA (final concentration). The isomerization of the Leu-Pro bond from cis to trans conformation catalysed by PPlase was measured by monitoring the change in the absorption at $330 \mathrm{~nm}$ using a Hitachi U-2010 UV/VIS spectrophotometer (Hitachi High-Technologies, Tokyo, Japan). The catalytic efficiency $\left(k_{\mathrm{cat}} / K_{\mathrm{M}}\right)$ was calculated from the relationship $k_{\text {cat }} / K_{M}=(\mathrm{kp}-\mathrm{kn}) / \mathrm{E}$, where $E$ represents the concentration of the enzyme, and $\mathrm{kp}$ and $\mathrm{kn}$ represent the first-order rate constants for the release of $p$-nitroaniline from the substrate in the presence and absence of the enzyme, respectively (Harrison \& Stein, 1990).

\section{Substrate Specificity}

Substrate specificity of Pk-FKBP35 was measured under protease-free and proteasecoupling assays using N-succinyl-Ala-Xaa-Pro-Phe-p-nitroanilide (Suc-AXaaPF-pNA) (Wako Pure Chemical, Osaka, Japan) as a substrate, in which Xaa stands for a variable aminoacyl residue in the $\mathrm{P} 1$ position of various substrates. The condition for proteasefree as described above. Meanwhile, the protease-coupling assay was performed according to Goh et al. (2018). The catalytic efficiency $\left(k_{\mathrm{cat}} / K_{\mathrm{M}}\right)$ obtained from SucAFPF-pNA was adjusted to $100 \%$ activity according to (Rahfeld et al., 1996; Budiman, Lindang, Cheong, \& Rodrigues, 2018). 


\section{RESULT AND DISCUSSION}

\section{Stability of Pk-FKBP35 towards Chymotrypsin}

Figure 1 shows 88 possible cleavage sites of chymotrypsin along the primary structure of Pk-FKBP35 predicted under the PeptideCutter (Gasteiger et al., 2005). This indicated that Pk-FKBP35 is prone to degradation of chymotrypsin as indicated by the presence of cleavage sites of the High- or Low-specificity of chymotrypsin along the sequences of Pk-FKBP35 in Figure 1. It is interesting that the cleavage sites are distributed in the whole region of Pk-FKBP35, extending from the N-terminal catalytic domain and C-terminal TPR domain. Nevertheless, a number of the cleavage sites at the catalytic domain was less than that of the TPR domain. This result implied that the presence of chymotrypsin in the protease-coupling assay would possibly degrade Pk-FKBP35. Nevertheless, the cleavage site predicted by the PeptideCutter is under the assumption that the cleavage sites are in exposed positions. In fact, in the folded state, those cleavage sites are possibly buried inside its three-dimensional structure and inaccessible by a protease to cleave. Parsell and Sauer (1989) demonstrated that the folded structure of protein is an important determinant for their proteolytic susceptibility. Ahmad, Kumar, Ramanand, and Rao (2012) proposed that partially or completely unfolded structures of the proteins are the major proteolytic susceptible conformations. Thomson, Hodgman, Yang, and Doyle (2003) also added that the PeptideCutter only relied on the presence of cleavage sites without taking into account cleavage rates affected by the structure of proteins. This leads to the assumption that the susceptibility of Pk-FKBP35 against chymotrypsin as predicted by the PeptideCutter might not reflect the natural environment where the protein is in a correct and completely folded structure. 

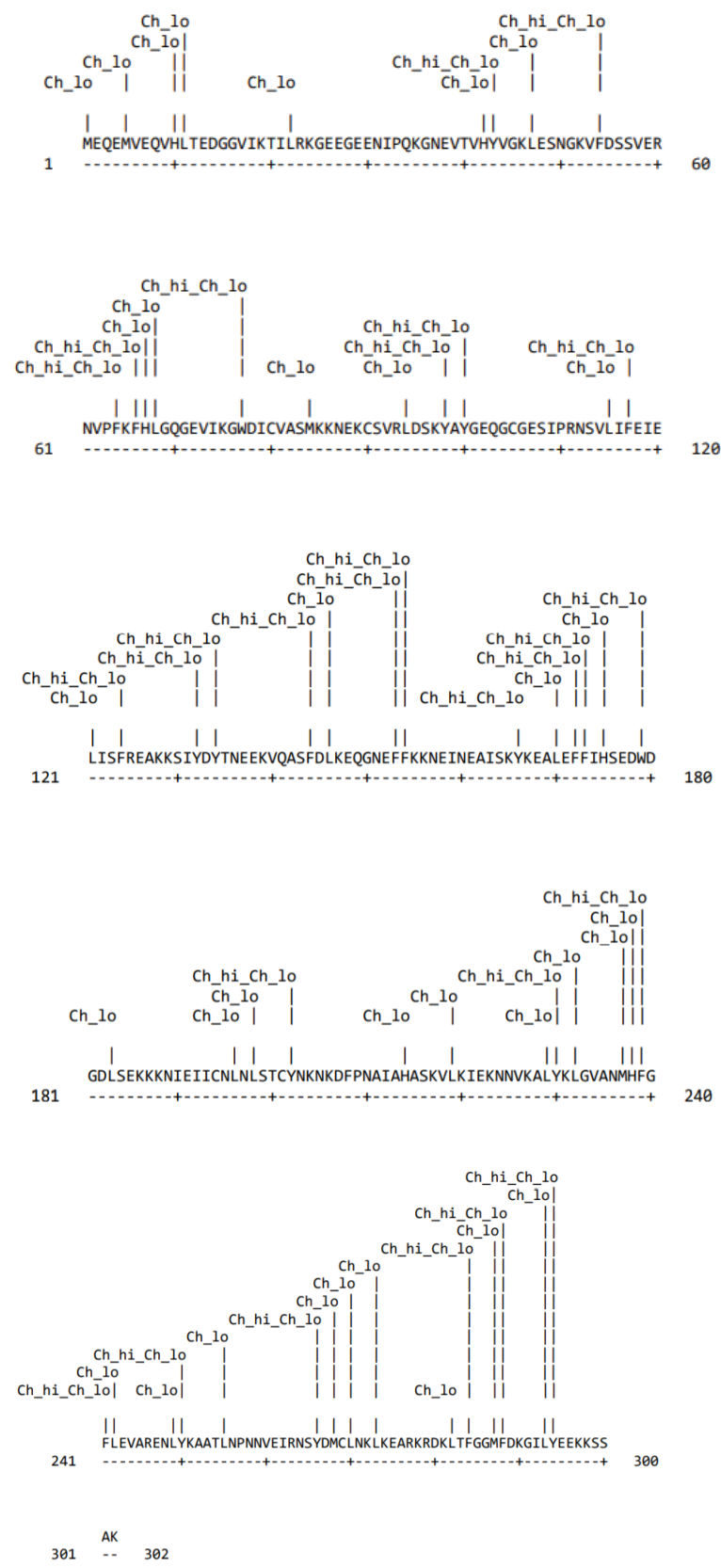

Figure 1 Theoretical cleavage sites of chymotrypsin on the primary sequence of Pk-FKBP35 predicted by the PeptideCutter. This diagram is derived by inputting amino acid sequence of Pk-FKBP35 into ExPASy PeptideCutter. The position of cleavage site is indicated by the dashed line above the sequence. Ch_lo and Ch_li represent the chymotrypsin-low specificity (C-term to [FYWML], not before P) and chymotrypsin-high specificity (C-term to [FYW], not before P), respectively. The numbers at the two ends of the sequence refer to the position of amino acids. 
To confirm this, a limited proteolysis assay was conducted experimentally where PkFKBP35 was challenged by chymotrypsin at various reaction times. The condition of the proteolysis was adjusted to mimic the protease-coupling assay. Figure 2 shows that Pk-FKBP35 was indeed degraded by chymotrypsin as indicated by the presence of some bands in addition to the bands corresponding to Pk-FKBP35 (38 kDa) and chymotrypsin $(25 \mathrm{kDa})$. Interestingly, degradation of Pk-FKBP35 by chymotrypsin resulted in only two major bands as shown in the Figure 2 as indicated by $B$ and $D$ bands. The apparent sizes of $B$ and $C$ bands were about 26 and $16 \mathrm{kDa}$, respectively, which are comparable to the sizes of TPR and catalytic domains of Pk-FKBP35, respectively. The $\mathrm{N}$-terminal amino acid sequencing on $\mathrm{D}$ band was found to be MGS_H_H, which corresponded to the His-tag tail located at N-terminal of the catalytic domain (Table 1). Meanwhile, the $\mathrm{N}$-terminal sequencing of band $\mathrm{B}$ produced two sequences of TNEE_V or EAK_SI (Table 1) corresponding to the residues located at the linker between the catalytic and TPR domain. This confirmed that $B$ and $D$ bands were indeed TPR and catalytic domains of Pk-FKBP35, respectively, which were separated upon degradation by chymotrypsin. This might also suggest that the major cleavage sites of chymotrypsin are located at the linker between these two domains. Earlier, the linker of the catalytic and TPR domains of Pk-FKBP35 structurally is a flexible loop with high solvent accessibility. A fragment between Ser113 and Gln 120 of the linker was found to have the highest flexibility and therefore predicted to be susceptible against protease (Silvester, Lindang, Chin, Ying, \& Budiman, 2017). Similarly, a multidomain FKBP22, which consisted of N-chaperone and C-catalytic domains, were also cleaved by chymotrypsin at the domain linkers (Budiman et al., 2012). The apparent stability of the functional domains of Pk-FKBP35 toward chymotrypsin was predicted due to the structures of the domains to bury the cleavage sites. This confirms that its folded form, the chymotrypsin cleavage sites on Pk-FKBP35 are much less than that of predicted by the PeptideCutter. 


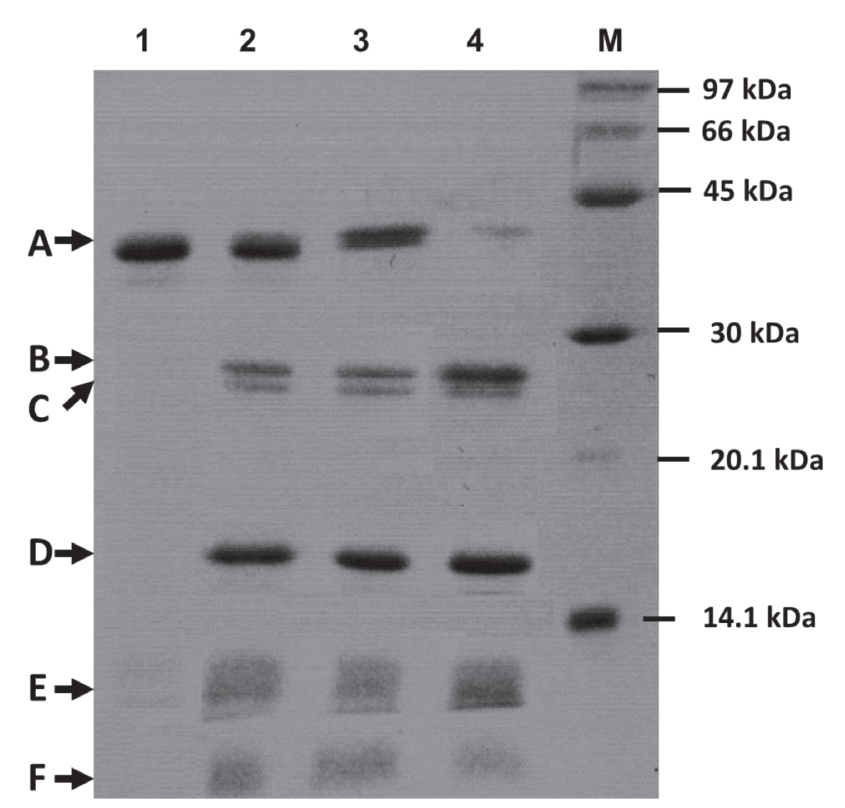

Figure 2 SDS-PAGE pattern of Pk-FKBP35 digestion by chymotrypsin. Lane 1 represented Pk-FKBP35 without chymotrypsin. Lanes 2, 3 and 4 represented Pk-FKBP35 in the presence of chymotrypsin after the incubation for 10,20 and $30 \mathrm{~min}$ at $25^{\circ} \mathrm{C}$, respectively. Lane $\mathrm{M}$ represented low molecular protein markers (GE Healthcare, Upsalla, Sweden). The protein bands corresponding to Pk-FKBP35 and chymotrypsin are indicated by $A$ and $C$ arrows, respectively. Meanwhile, B, D, E and F arrows indicated the digestion products.

Table 1 The N-terminal sequences of the protein bands from limited proteolysis

$\begin{array}{cl}\text { Band } & \text { Sequence } \\ \text { A } & \text { MGSS_HH } \\ \text { B } & \text { TNEE_V } \\ & \text { EAK_SI } \\ \text { D } & \text { MGS_H_H } \\ \text { E } & \text { Unable to be sequenced } \\ \text { F } & \text { Unable to be sequenced } \\ \text { \#"_" indicated the residue is unable to read due to low signal. }\end{array}$


Interestingly, the band corresponding to the catalytic domain ( $D$ band) was relatively stable up to 30 min reaction time. Meanwhile, the band's thickness corresponds to the TPR domain was shown to decrease during the proteolytic time. These indicated that the catalytic domain was relatively much more stable than the TPR domain. The decrease in the band of the TPR domain indicated that this protein was further digested by chymotrypsin. To note, some minor fragments were also observed in Figure 2 (labelled by $\mathrm{E}$ and $\mathrm{F}$ arrows) which indicated that the chymotrypsin also able to cleave some fragments of Pk-FKBP35 apart from the linker. Nevertheless, these fragments are considered minor degradation products and might be resulted from the degradation of the surface region of this protein. Altogether, the digestion of PkFKBP35 by chymotrypsin occurred primarily at the domain linker producing free forms of the catalytic and TPR domains. The chymotrypsin further digested the TPR domain producing smaller fragments ( $E$ and $F$ bands). Further digestion might also occurred in the catalytic domain, yet, at a much lower rate than the TPR domain. Structurally, the catalytic domain folded into a more globular structure, while the TPR domain folded into a more elongated structure (Silvester et al., 2017; Alag et al., 2009), which made this domain is theoretically more prone to proteolytic degradation. Unfortunately, the $\mathrm{N}$-terminal sequencings on $\mathrm{E}$ and $\mathrm{F}$ bands were unsuccessful (Table 1), which might be due to various fragments accumulated in these bands.

To note, despite this protein is degradable by chymotrypsin, remarkable catalysis of slow cis prolyl bond isomerization by Pk-FKBP35 was observed (Goh et al., 2018). This indicated that the cleavage of Pk-FKBP35 by chymotrypsin was not in the region responsible for catalysis. The domain linker of Pk-FKBP35 was indeed found not to play any role in catalysis (Silvester et al., 2017). Similarly, the TPR domain, which was apparently sensitive toward chymotrypsin, was not involved in the catalytic activity of Pk-FKBP35. Besides, the isolated catalytic domain (without TPR domain) remains exhibit comparable catalytic activity to its full-length (Goh et al., 2018).

\section{Catalytic Activity}

Figure 3 shows that the time course of reversible prolyl isomerization of Suc-AlaPhe-Ala-pNA upon the solvent jumping in the presence of $30 \mathrm{nM}$ of Pk-FKBP35 or D55A active site mutant, was found to be considerably different to the spontaneous isomerization reaction. While the isomerization time courses as shown in Figure 3 were found to have scattered light noise appearance, the courses were well first-order fitted with $\mathrm{R}^{2}$ more than $95 \%$ for both reactions. The first-order reaction rate $(k)$ of the cis-trans isomerization in the absence of Pk-FKBP35 was calculated to be $4.0 \pm 0.81 \times$ $10^{-3} \mathrm{~s}^{-1}$, which is comparable to the previous report on the isomerization rate constant in the absence of PPlase (Janowski et al., 1997; Schiene-Fischer, Habazetti, Tradler, \& 
Fischer, 2002; Fanghanel \& Fischer, 2004; Kofron et al., 1992). Interestingly, Goh et al. (2018) found that under protease-coupling assay, the $k$ value of the isomerization in the absence of Pk-FKBP35 was in the range $3.70-4.52 \times 10^{-3} \mathrm{~s}^{-1}$, which is close enough to the $k$-value obtained in this study. This suggested that the presence of chymotrypsin has no serious effect on the isomerization rate of the substrate.

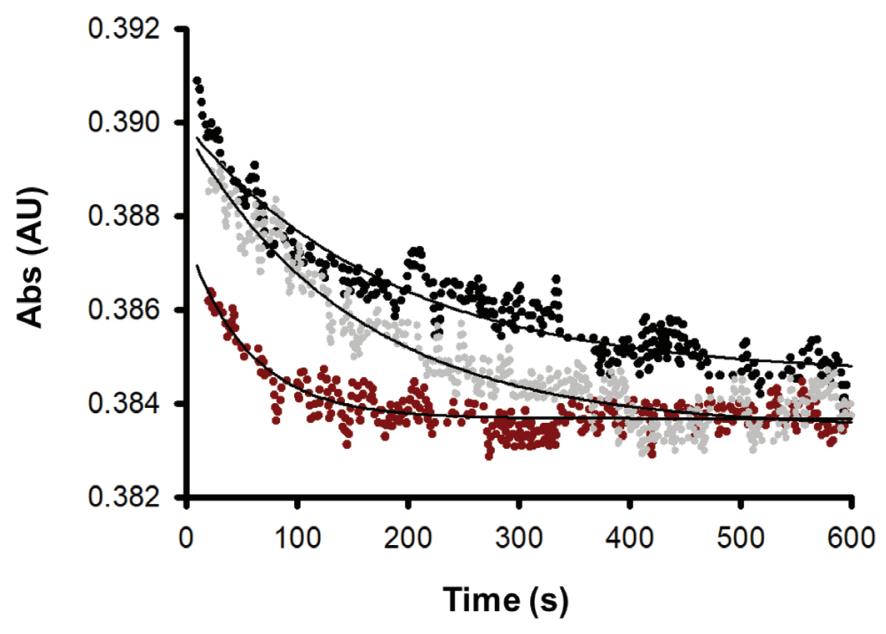

Figure 3 Progress curves of the prolyl cis-trans isomerization of Suc-AlaPhe-Pro-Phe-4-nitroanilide after jumping from the peptide stock solution in TFE/LiCl into the final buffer solution in the absence (black circle) or in the presence of Pk-FKBP35 (red circle) or D55A mutant (grey circle). The solid black line represents the fitting curve of a first order-rate reaction for each curve.

Further, Figure 4 indicated that the $k$ value of the isomerization rate in the presence of Pk-FKBP35 was found to be concentration-dependent, which follows the general trend of PPlase catalytic activity (Fanghanel \& Fischer, 2004; Schiene-Fischer et al., 2002). Earlier, Goh et al. (2018) also found that the PPlase activity of Pk-FKBP35 measured under protease-coupling assay was a concentration-dependent. The calculated $k_{\text {cat }} /$ $K_{\mathrm{M}}$ from this was found to be $4.5 \pm 0.13 \times 10^{5} \mathrm{M}^{-1} \mathrm{~s}^{-1}$, which is remarkable enough to demonstrate the ability of this protein to catalyse the slow cis-trans isomerization. Nevertheless, Goh et al. (2018) reported that $k_{\text {cat }} / K_{\mathrm{M}}$ of Pk-FKBP35 under proteasecoupling assay was found to be slightly higher $\left(5.0 \pm 0.18 \times 10^{5} \mathrm{M}^{-1} \mathrm{~s}^{-1}\right)$. This might be due to differences in the structural freedom of the catalytic domain of Pk-FKBP35 in the absence or in the presence of chymotrypsin. In the protease-coupling assay, chymotrypsin likely digested the linker of catalytic and TPR domain yielding a free catalytic domain in the assay cocktail. In the absence of chymotrypsin, the catalytic domain remains in its intact form with the TPR domain in a dimeric form. Structurally, the catalytic domain is more restricted due to the dimerization of this protein 
as previously reported (Goh et al., 2018). The free forms of the catalytic domain of Pk-FKBP35 are predicted to have more flexibility to bind to more substrate which consequently exhibiting higher catalytic activity than in the dimeric form. Similarly, Budiman et al. (2009) experimentally confirmed that the monomeric catalytic domain of FKBP was found to be more active than its dimeric form due to the less structural constraints to bind to the substrate.

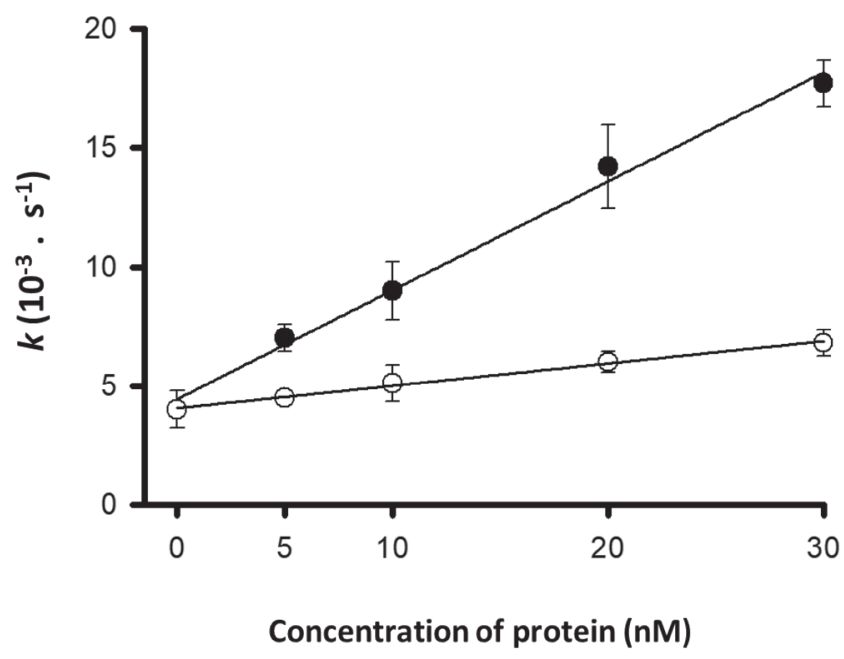

Figure 4 The first-order reaction rates $(k)$ of the prolyl cis-trans isomerization in the presence of a various concentrations of Pk-FKBP35 (black circle) and D55A mutant (white circle)

To note, the $k_{\text {cat }} / K_{M}$ values for Pk-FKBP35 obtained from protease-free and proteasecoupling assays were different by less than $10 \%$. This magnitude is considered minor for enzymatic activity (Bisswanger, 2014), and therefore we considered that the catalytic activity of Pk-FKBP35 measured using both assays was comparable. Further, the $k_{\text {cat }} / K_{\mathrm{M}}$ of D55A mutant under the protease-free assay was calculated to be 0.81 $\pm 0.05 \times 10^{5} \mathrm{M}^{-1} \mathrm{~s}^{-1}$. This value is considerably comparable to the previous report by Goh et al. (2018) showing that the D55A mutant of Pk-FKBP35 exhibited $k_{\text {cat }} / K_{\mathrm{M}}$ of 1.01 $\pm 0.14 \times 10^{5} \mathrm{M}^{-1} \mathrm{~s}^{-1}$ under a protease-coupling method. Low activity of D55A mutant was previously proposed due to the possible role of this residue to reduce a resonance effect of the peptide bond through the formation of a hydrogen bond to the peptide bond nitrogen (Goh et al., 2018). Fanghanel and Fisher (2004) proposed earlier that disrupting the resonance of a peptide bond is an essential step in the acceleration of the isomerization of the bond. The resonance structure caused the bond is structurally rigid and has restricted rotation. 
As the $k_{\text {cat }} / K_{\mathrm{M}}$ values of Pk-FKBP35 and D55A mutant measured under protease-free and protease-coupling assays were comparable, we, therefore, assumed that that protease-coupling assay is considered to be reliable for measuring the catalytic activity of Pk-FKBP35. In fact, as Figure 2 obviously showed catalytic domain of PkFKBP35 is relatively stable against chymotrypsin which leads to an assumption that the essential region for catalytic activity was not interfered by chymotrypsin. In addition, the stability of both domains might also due to the low concentration of chymotrypsin used in the protease-coupling assay was relatively low. Noteworthy, the protease-free assay was found to have less signal-to-noise ratio as indicated in Figure 3. Janowski et al. (1997) and Kofron et al. (1992) indicated that the high spectroscopic noise is due to small differences in spectroscopic absorbance between trans and cis prolyl bond isomers. In combination with the apparent stability of the catalytic domain of Pk-FKBP35 against chymotrypsin and the spectroscopic noise, we are in the opinion that the protease-coupling assay is acceptable and preferable for assessment of catalytic Pk-FKBP35. Fanghanel and Fischer (2004) also indicated that the proteasecoupling assay is experimentally proven and scientifically acceptable for many types of PPlase. The protease-free assay is, nevertheless, recommended for PPlase members having low stability against chymotrypsin. In addition, to address the noise observed in UV-vis spectroscopy, NMR spectroscopy is more preferable for assessing the direct isomerization rate of the prolyl bond under protease-free assay.

\section{Substrate Specificity}

A tetrapeptide substrate containing different amino acid preceding proline residue (Suc-Ala-Xaa-Pro-Pro-pNA) was used to determine the substrate specificity of PkFKBP22. Figure 5 indicated the position of the Xaa residue which refers to the P1 position in the nomenclature according to the Schechter and Berger nomenclature (Berger \& Schechter, 1970). Based on the nomenclature, the residues involved in the catalytic activity of Pk-FKBP35 (active site residues) were assumed to form a contiguous pocket termed subsite. Each subsite (S2, S1, S1' and S2') represents a single active site residue of Pk-FKBP35 that binds to corresponding residues of the peptide substrate (P2, $\mathrm{P} 1, \mathrm{P} 1^{\prime}$ and $\left.\mathrm{P} 2^{\prime}\right)$. The bond to be isomerized (prolyl-bond) is located between P1 - P1' sites of the substrate. Figure 5 showed the subsites S2, S1, $\mathrm{S}^{\prime}$ and $\mathrm{S} 2$ ' would interact to $\mathrm{P} 2, \mathrm{P} 1, \mathrm{P} 1$ ' and $\mathrm{P} 2$ ', respectively. Any changes on the $\mathrm{P} 2$, $\mathrm{P} 1, \mathrm{P} 1^{\prime}$ and $\mathrm{P} 2$ ' sequence might affect the interaction with the subsites. In this study, eleven (11) variants of the tetrapeptide substrates, with different chemical properties and bulkiness, were used by replacing Xaa to Leu/Phe/Trp/Ala/GIn/Val/Ile/His/Asp/ Lys/Glu. Schiene, Reimer, Schutkowski, and Fischer (1998) reported that the residue prior to the proline was found to be critical for spontaneous bond rotation due to its role in formation of the non-covalent enzyme/substrate complex. 


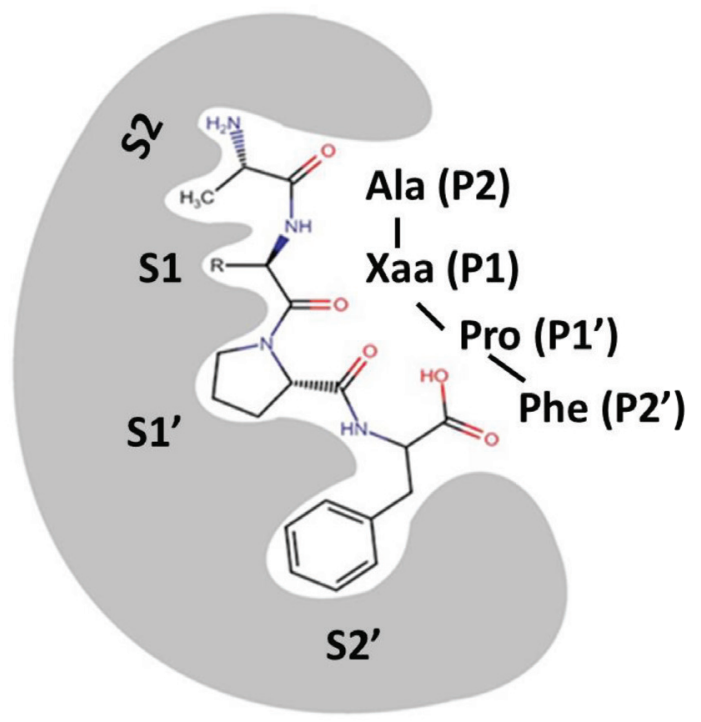

Figure 5 Schematic and nomenclature for binding of the peptide substrate to Pk-FKBP35 according to the Schechter and Berger nomenclature for binding of a peptide substrate to a peptidase. Pk-FKBP35 is represented by the shaded area. $\mathrm{R}$ is the side chain of Xaa amino acid. P2, P1, P1' and P2' are the amino acid residues of the substrate. S2, S1, S1' and S2' are the corresponding subsites on Pk-FKBP35. The figure is not drawn to scale.

Figure 6 shows that the overall preference of Pk-FKBP35 to Xaa is more to the hydrophobic residues. The highest catalytic efficiency $\left(k_{\text {cat }} / K_{M}\right)$ was observed for Leu at Xaa position. Meanwhile, no activity was detected when Glu was at Xaa site. In overall, the preference of Pk-FKBP35 towards Xaa at P1 position followed the following order: Leu $>$ Phe $>$ Lys $>$ Trp $>$ Val $>$ lle $>$ His $>$ Asp $>$ Ala $>$ Gln $>$ Glu. This suggested that Pk-FKBP35 behaves similarly as other FKBP family proteins in which hydrophobic residues as a residue preceding the proline (Fanghanel \& Fischer, 2004; Rahfeld et al., 1996; Budiman et al., 2018). Interestingly, while lle and Val are considerably more hydrophobic than Leu, Phe and Trp, based on their hydropathy index (Kyte \& Doolittle, 1983), the activity against lle or Val was much lower than that of Leu or Phe or Trp. This might be due to the stereochemical properties of Val or lle side chains which are not appropriate for $\mathrm{S} 1$ site. The side chain of lle is believed to be too bulky to fit the S1 site, while Val's side chain is too short and branched thus unable to interact to the $\mathrm{S} 1$ site properly. A similar explanation also applied for Ala, where its single methyl side chain is not sufficient to interact property to the $\mathrm{S} 1$ site. Besides, study on human FKBP12 by Schiene et al. (1998) indicated that the methyl group of Ala's side chain was at opposite orientation of the S1 site. Interestingly, while Lys is considered as a hydrophilic residue, the activity against this residue was higher than some hydrophobic residues (Trp, Val, lle). It is believed that this due to the hydrophobicity of the carbon atom $(\mathrm{Ca}-\mathrm{C \varepsilon})$ of this residue that fit well the S1 site as also supported by Fanghanel \& Fischer (2004). 


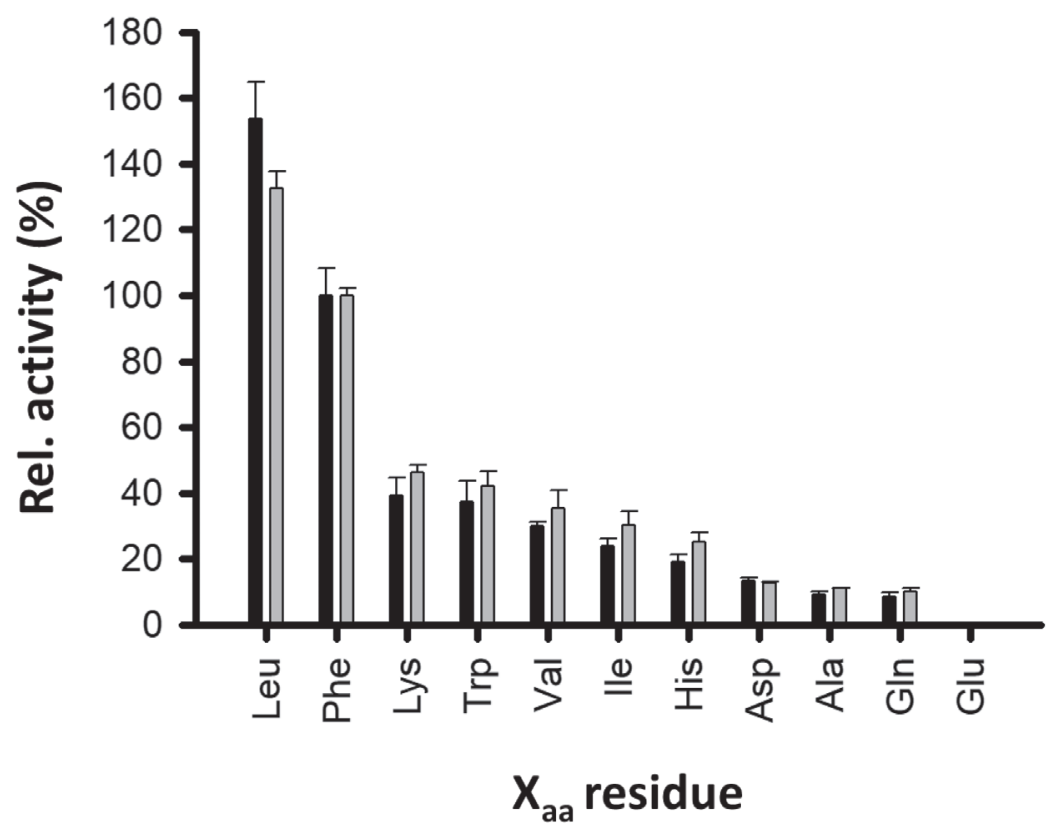

Figure 6 Substrate specificity of Pk-FKBP35 as measured under protease-free (black bars) and proteasecoupling (grey bars) assays. The PPlase activity towards Phe as the Xaa residue in the substrate was adjusted to $100 \%$ activity.

To note, the catalytic domain of Pk-FKBP35 displays high similarity to the other FKBPs members (Goh et al., 2018) assuming that the proteins share the substrate binding and catalytic mechanisms. Nevertheless, the uniqueness of Pk-FKBP35 lies in the loop corresponding to the loop 80's of human FKBP12, whereby some residues of the FKBP35's loop were found to be different from that of human FKBP12. Nevertheless, the low conservation on the loop apparently has no serious effect on the substrate specificity as human FKBP12 was also reported to prefer bulky hydrophobic residues at Xaa position (Ikura \& Ito, 2007). Meanwhile, the preferences of cyclophilin and parvulin toward the Xaa position were reported to be different. Cyclophilin has wide specificity at Xaa position (Rahfeld et al., 1994), while Parvulin having a unique specificity for a phosphorylated Ser or Thr preceding the Pro (Fanghanel \& Fischer, 2004; Rahfeld et al., 1994). The discrepancy in the specificity among PPlase member is believed due to structural differences in substrate binding pocket that affect the fitting of each substrate (Fanghanel \& Fischer, 2004).

Interestingly, when the protease-coupling assay was used to measure the substrate specificity, the preferences of the Xaa residues were also similar to that of the proteasefree assay. This indicated that the presence of chymotrypsin in the assay has no serious effect on the substrate fitting into the catalytic activity. This supports our assumption earlier that the catalytic domain was relatively resistant towards chymotrypsin under 
assay condition. Noteworthy, the catalytic domain of Pk-FKBP35 is assumed to be in free monomeric form due to the digestion of the domain linker. Meanwhile, the catalytic domain is in an intact dimeric form when the chymotrypsin is an absence in the assay. This suggested that the substrate specificity of this protein is independent from its dimerization. However, as the catalytic efficiency of Pk-FKBP35 deducted from the protease-coupling assay was slightly higher than that of from the proteasefree assay, this also suggests that there is no structural relationship between catalytic efficiency and substrate specificity. Catalytic efficiency might be more related to the flexibility of overall the catalytic domain, located at the tip of the dimeric structure of Pk-FKBP35, in capturing the substrate. Structural dimerization might affect the binding constant $\left(k_{\text {on }}\right.$ or $\left.k_{\text {off }}\right)$ to the catalytic domain. Specificity was then speculated to be more related to the local structural configuration at the active site. Similar evidence was also reported for the dimeric form of FKBP22 from Shewanella sp. SIB1 (Budiman et al., 2009; Budiman et al., 2018). Inoue et al. (2019) and Parker, Corey, Stansfeld, and Newstead (2019) also implied that structural basis regulating the substrate specificity heavily relied on the local configuration of the substrate-binding cavity.

\section{CONCLUSION}

The current study confirmed the structural sensitivity of Pk-FKBP22 towards chymotrypsin, particularly the linker connecting catalytic and dimerization domains of this protein. Nevertheless, the disruption of the linker by chymotrypsin showed no apparent effect on its catalytic properties. This is based on the results that the catalytic activity of Pk-FKBP35 assessed using protease-coupling and protease-free assays were comparable. In addition, the substrate specificity of Pk-FKBP35 towards Xaa residues at the $\mathrm{P} 1$ position measured using protease-coupling and protease-free assays also revealed the same substrate pattern, whereby the hydrophobic bulky residues are preferable at Xaa site. This confirmed that both protease-coupling and proteasefree assays are reliable enough to assess the catalytic properties of Pk-FKBP35. Nevertheless, due to the high spectrophotometric noise issue under protease-free assay, the protease-coupling is believed to be practically more preferable for the assay.

\section{ACKNOWLEDGEMENTS}

This project is supported by the Skim Penyelidikan Berimpak, Universiti Malaysia Sabah (SPB0003-2020) and the International Collaborative Grant of Protein Research Institute, Osaka University, Japan 2019 (TLA1902). The authors would like to thank Dr Takashi Kodama for his technical support. 


\section{REFERENCES}

Ahmad, S., Kumar, V., Ramanand, B., \& Rao, N. M. (2011). Probing protein stability and proteolytic resistance by loop scanning: A comprehensive mutational analysis. Protein Science, 21, 433 - 446. DOI: 10.1002/pro.2029

Alag, R., Bharatham, N., Dong, A., Hills, T., Harikishore, A., Widjaja, A.A, ... Yoon, H. S, (2009). Crystallographic structure of the tetratricopeptide repeat domain of Plasmodium falciparum FKBP35 and its molecular interaction with $\mathrm{Hsp90}$ C-terminal pentapeptide. Protein Science: a Publication of the Protein Society, 18 (10), 2115 - 2124. DOI: 10.1002/ pro.226.

Berger, A., \& Schechter, I. (1970). Mapping the active site of papain with the aid of peptide substrates and inhibitors. Philosophical transactions of the Royal Society of London. Series B, Biological Sciences, 257 (813), 249 - 264. DOI: 10.1098/rstb.1970.0024

Bisswanger, H. (2014). Enzyme assays. Perspectives in Science, 1, 41 - 55. DOI: http://dx.doi. org/10.1016/j.pisc.2014.02.005

Budiman, C., Bando, K., Angkawidjaja, C., Koga, Y., Takano, K., \& Kanaya, S. (2009). Engineering of monomeric FK506-binding protein 22 with peptidyl prolyl cis-trans isomerase. FEBS Journal, 276 (15), 4091 - 4101. DOI: https://doi.org/10.1111/j.1742-4658.2009.07116.x

Budiman, C., Lindang, H. U., Cheong, B. E., \& Rodrigues, K. F. (2018). Inhibition and substrate specificity properties of FKBP22 from a psychrotrophic bacterium, Shewanella sp. SIB1. Protein Journal, 37 (3), 270 - 279. DOI: https://doi.org/10.1007/s10930-018-9772-z

Budiman, C., Tadokoro, T., Angkawidjaja, C., Koga, Y., \& Kanaya, S. (2012). Role of polar and nonpolar residues at the active site for PPlase activity of FKBP22 from Shewanella sp. SIB1. FEBS Journal, 279 (6), 976 - 986. DOI: 10.1111/j.1742-4658.2012.08483.x

Divis, P. C., Hu, T. H., Kadir, K. A., Mohammad, D., Hii, K.C., Daneshvar, C., ... Singh, B. (2020). Efficient surveillance of Plasmodium knowlesi genetic subpopulations, Malaysian Borneo, 2000-2018. Emerging Infectious Diseases, 26 (7), 1392 - 1398. DOI: https:// dx.doi.org/10.3201/eid2607.190924

Fanghanel, J., \& Fischer, G. (2004). Insight into catalytic mechanism of peptidyl prolyl cis/trans isomerase. Frontiers Bioscience, 9, 3453 - 3478. DOI: 10.2741/1494.

Gasteiger, E., Hoogland, C., Gattiker, A., Duvaud, S., Wilkins, M.R., Appel, R. D., \& Bairoch, A. (2005). Protein identification and analysis tools on the ExPASy server. In J. M. Walker (Ed.), The proteomics protocols handbook (pp. 571 - 607). Totowa, New Jersey: Humana Press.

Goh, C. K. W., Silvester, J., Wan Mahadi, W. N. S., Lee, P. C., Lau, T. Y., Thean, C. L., ... Budiman, C. (2018). Expression and characterization of functional domains of FK506-binding protein 35 from Plasmodium knowlesi. Protein Engineering, Design and Selection, 31 (12), 489 - 498. DOI: 10.1093/protein/gzz008

Goh, X. T., Lim, Y. A., Vythilingam, I., Chew, C. H., Lee, P. C., Tan, T. C., ... Chua, K. H. (2013). Increased detection of Plasmodium knowlesi in Sandakan division, Sabah as revealed by PlasmoNex ${ }^{\text {TM }}$. Malaria Journal, 12, 264. DOI: https://doi.org/10.1186/1475-2875$12-264$

Goodwin, T. W., \& Morton, R. A. (1946) The spectrophotometric determination of tyrosine and tryptophan in proteins. Biochemical Journal, 40 (5 - 6), 628 - 632. DOI: 10.1042/ bj0400628

Harikishore, A., Niang, M., Rajan, S., Preiser, P. R., \& Yoon, H. S. (2013). Small molecule Plasmodium FKBP35 inhibitor as a potential antimalaria agent. Scientific Reports, 3, 2501. DOI: https://doi.org/10.1038/srep02501 
Ikura, T., \& Ito, N. (2007). Requirements for peptidyl-prolyl isomerization activity: a comprehensive mutational analysis of the substrate-binding cavity of FK506-binding protein 12. Protein Science, 16 (12), 2618 - 2625. DOI: 10.1110/ps.073203707

Inoue, Y., Ogawa, Y., Kinoshita, M., Terahara, N., Shimada, M., Kodera, N., .. Minamino, T. (2019). Structural insights into the substrate specificity switch mechanism of the type III protein export apparatus. Structure, 27 (6), 965 - 976. DOI: 10.1016/j.str.2019.03.017

Janowski, B., Wöllner, S., Schutkowski, M., \& Fischer, G. (1997). A protease-free assay for peptidyl prolyl cis/trans isomerases using standard peptide substrates. Analytical Biochememistry, 252 (2), 299 - 307. DOI: 10.1006/abio.1997.2330

Kyte, J., \& Doolittle, R. F. (1983). A simple method for displaying the hydropathic character of a protein. Journal of Molecular Biology, 157 (1), 105 - 132.

Laemmli, U. K. (1970). Cleavage of structural proteins during the assembly of the head of bacteriophage. Nature, 227, $680-685$.

Lau, T. Y., Joveen-Neoh, W. F., \& Chong, K. L. (2011). High Incidence of Plasmodium knowlesi Infection in the Interior Division of Sabah, Malaysian Borneo. International Journal of Bioscience, Biochemistry and Bioinformatics, 1 (2), 163 - 167. DOI: 10.7763/IJBBB.2011. V1.30

Kofron, J. L., Kuzmic, P., Kishore, V., Gemmecker, G., Fesik, S. W., \& Rich, D. H. (1992). Lithium chloride perturbation of cis-trans peptide bond equilibria: effect on conformational equilibria in cyclosporin A and on time-dependent inhibition of cyclophilin. Journal of the American Chemical Society, 114 (7), 2670 - 2675. DOI: https://doi.org/10.1021/ ja00033a047

Parker, J. L., Corey, R. A., Stansfeld, P. J., \& Newstead, S. (2019). Structural basis for substrate specificity and regulation of nucleotide sugar transporters in the lipid bilayer. Nature Communications, 10, 4657. DOI: https://doi.org/10.1038/s41467-019-12673-w

Parsell, D. A., \& Sauer, R.T. (1989). The structural stability of a protein is an important determinant of its proteolytic susceptibility in Escherichia coli. The Journal of Biological Chemistry, 264 (13), $7590-7595$.

Rahfeld, J. U., Rucknagel, K. P., Schelbert, B., Ludwig, B., Hacker, J., Mann, K., \& Fischer, G. (1994). Confirmation of the existence of a third family among peptidyl-prolyl cis/trans isomerases. Amino acid sequence and recombinant production of parvulin. FEBS Letters, 352 (2), 180 - 184. DOI: https://doi.org/10.1016/0014-5793(94)00932-5

Rahfeld, J. U., Rucknagel, K. P., Stoller, G., Horne, S. M., Schierhorn, A., Young, K. D., \& Fischer, G. (1996). Isolation and amino acid sequence of a new 22-kDa FKBP-like peptidyl-prolyl cis/trans-isomerase of Escherichia coli similarity to Mip-like proteins of pathogenic bacteria. The Journal of Biological Chemistry, 271 (36), 22130 - 22138.

Rajharam, G. S., Cooper, D. J., William, T., Grigg, M. J., Anstey, N. M., \& Barber, B. E. (2019). Deaths from Plasmodium knowlesi Malaria: Case series and systematic review. Clinical Infectious Diseases, 69 (10), 1703 - 1711. DOI: 10.1093/cid/ciz011

Schiene, C., Reimer, U., Schutkowski, M., \& Fischer, G. (1998). Mapping the stereospecificity of peptidyl prolyl cis/trans isomerases. FEBS Letter, 432 (3), 202 - 206. DOI: 10.1016/ s0014-5793(98)00871-0

Schiene-fischer, C., Habazetti, J., Tradler, T., \& Fischer, G. (2002). Evaluation of similarities in the cis/trans isomerase function of trigger factor and DnaK. Biological Chemistry, 383, 1865 - 1873. DOI: 10.1515/BC.2002.210 
Silvester, J., Lindang, H. U., Chin, L. P., Ying, L. T., \& Budiman, C. (2017). Structure and molecular dynamic regulation of FKBP35 from Plasmodium knowlesi by structural homology modeling and electron microscopy. Journal of Biological Sciences, 17 (8), $369-380$. DOI: 10.3923/jbs.2017.369.380

Singh, B., \& Daneshvar, C. (2013). Human infections and detection of Plasmodium knowlesi. Clinical Microbiology Reviews, 26 (2), 165 - 184. DOI: 10.1128/CMR.00079-12

Singh, B., Sung, L. K., Matusop, A., Radhakrishnan, A., Shamsul, S. S. G., Cox-Singh, J., ... Conway, D. J. (2004). A large focus of naturally acquired Plasmodium knowlesi infections in human beings. Lancet, 363 (9414), 1017 - 1024. DOI: 10.1016/S0140-6736(04)15836-4

Thomson, R., Hodgman, T. C., Yang, Z. R., \& Doyle, A. K. (2003). Characterizing proteolytic cleavage site activity using bio-basis function neural networks. Bioinformatics, 19 (14), 1741 - 1747. DOI: 10.1093/bioinformatics/btg237

William, T., Jelip, J., Menon, J., Anderios, F., Mohammad, R., Mohammad, T. A. A., ... Barber, B. E. (2014). Changing epidemiology of malaria in Sabah, Malaysia: increasing incidence of Plasmodium knowlesi. Malaria Journal, 13 (1), 390. DOI: https://doi.org/10.1186/14752875-13-390

Yoon, H. R., Kang, C. B., Chia, J., Tang, K., \& Yoon, H. S. (2006). Expression, purification, and molecular characterization of Plasmodium falciparum FK506-binding protein 35 (PfFKBP35). Protein Expression and Purification, 53 (1), 179 - 185. DOI: https://doi. org/10.1016/j.pep.2006.12.019 
